\title{
Comparative transcriptomics reveals PrrAB- mediated control of metabolic, respiration, energy-generating, and dormancy pathways in Mycobacterium smegmatis
}

Jason D. Maarsingh ${ }^{1,2}$, Shanshan Yang ${ }^{3}$, Jin G. Park ${ }^{4}$ and Shelley E. Haydel ${ }^{1,5^{*}}$ [D

\begin{abstract}
Background: Mycobacterium smegmatis is a saprophytic bacterium frequently used as a genetic surrogate to study pathogenic Mycobacterium tuberculosis. The PrrAB two-component genetic regulatory system is essential in M. tuberculosis and represents an attractive therapeutic target. In this study, transcriptomic analysis (RNA-seq) of an M. smegmatis $\triangle p r r A B$ mutant was used to define the PrrAB regulon and provide insights into the essential nature of PrrAB in M. tuberculosis.
\end{abstract}

Results: RNA-seq differential expression analysis of M. smegmatis wild-type (WT), $\triangle p r r A B$ mutant, and complementation strains revealed that during in vitro exponential growth, PrrAB regulates 167 genes $(q<0.05)$, $57 \%$ of which are induced in the WT background. Gene ontology and cluster of orthologous groups analyses showed that PrrAB regulates genes participating in ion homeostasis, redox balance, metabolism, and energy production. PrrAB induced transcription of $\operatorname{dos} R(\operatorname{dev} R)$, a response regulator gene that promotes latent infection in M. tuberculosis and 21 of the $25 \mathrm{M}$. smegmatis DosRS regulon homologues. Compared to the WT and complementation strains, the $\triangle p r r A B$ mutant exhibited an exaggerated delayed growth phenotype upon exposure to potassium cyanide and respiratory inhibition. Gene expression profiling correlated with these growth deficiency results, revealing that PrAB induces transcription of the high-affinity cytochrome bd oxidase genes under both aerobic and hypoxic conditions. ATP synthesis was 64\% lower in the $\triangle p r r A B$ mutant relative to the WT strain, further demonstrating that PrrAB regulates energy production.

Conclusions: The M. smegmatis PrrAB two-component system regulates respiratory and oxidative phosphorylation pathways, potentially to provide tolerance against the dynamic environmental conditions experienced in its natural ecological niche. PrrAB positively regulates ATP levels during exponential growth, presumably through transcriptional activation of both terminal respiratory branches (cytochrome $c b c_{1}-a a_{3}$ and cytochrome $b d$ oxidases), despite transcriptional repression of ATP synthase genes. Additionally, PrrAB positively regulates expression of the dormancy-associated dos $R$ response regulator genes in an oxygen-independent manner, which may serve to finetune sensory perception of environmental stimuli associated with metabolic repression.

Keywords: Mycobacterium smegmatis, Mycobacterium tuberculosis, prrAB, Two-component system, RNA-seq, Transcriptomics, Hypoxia, Respiration, Oxidative phosphorylation, ATP

\footnotetext{
* Correspondence: Shelley.Haydel@asu.edu

${ }^{1}$ School of Life Sciences, Arizona State University, Tempe, AZ, USA

${ }^{5}$ The Biodesign Institute Center for Immunotherapy, Vaccines and

Virotherapy, Arizona State University, Tempe, AZ, USA

Full list of author information is available at the end of the article
}

(c) The Author(s). 2019, corrected publication 2019. Open Access This article is distributed under the terms of the Creative Commons Attribution 4.0 International License (http://creativecommons.org/licenses/by/4.0/), which permits unrestricted use, distribution, and reproduction in any medium, provided you give appropriate credit to the original author(s) and the source, provide a link to the Creative Commons license, and indicate if changes were made. The Creative Commons Public Domain Dedication waiver (http://creativecommons.org/publicdomain/zero/1.0/) applies to the data made available in this article, unless otherwise stated. 


\section{Background}

Two-component systems (TCSs) participate in signal transduction pathways and are ubiquitously found in bacteria, archaea, some lower eukaryotes and plants [1-4]. TCSs recognize specific environmental stimuli [5] and integrate an adaptive response, frequently by modulating transcription [6]. A prototypical TCS consists of a membrane-bound histidine kinase sensor and a cytoplasmic DNA-binding response regulator. In pathogenic bacteria, TCSs act as virulence factors that regulate diverse survival mechanisms, such as antibiotic resistance [7], phosphate limitation [8], low oxygen tension [9], and evasion of immune responses [10]. Though mammalian proteins bearing histidine kinase sequence motifs and activity [11] have been identified, response regulators appear absent in humans, opening the possibility for development of inhibitors targeting virulence-related or essential bacterial TCSs as novel therapeutic approaches.

Mycobacterium tuberculosis, the causative agent of tuberculosis, is an ancient disease of mankind and the leading cause of death from an infectious agent [12]. The M. tuberculosis genome harbors 11 paired TCSs, two orphaned histidine kinases, and six orphaned response regulators [13]. Of these TCSs, only MtrAB [14] and PrAB [15] are essential for $M$. tuberculosis viability. The $\operatorname{prr} A$ response regulator and $\operatorname{prr} B$ histidine kinase genes are conserved across all fully-sequenced mycobacterial genomes, suggesting an evolutionary selective pressure to retain these TCS genes. M. tuberculosis prrAB is upregulated during the early stages of human macrophage infection [13] and under in vitro nitrogen limitation [15]. During infection in murine macrophages, $\operatorname{prr} A B$ is required for early replication and adaptation to the intracellular environment [16]. Capitalizing on findings that diarylthiazole compounds inhibit $M$. tuberculosis growth via the PrrAB TCS, Bellale et al. [17] exposed $M$. tuberculosis cultures to diarylthiazole and found that PrAB modulates transcription of genes enabling metabolic adaptation to a lipid-rich environment, responsiveness to reduced oxygen tension, and production of essential ribosomal proteins and amino acid tRNA synthases.

Mycobacterium smegmatis strain $\mathrm{mc}^{2} 155$ [18] is a non-pathogenic, rapid-growing, saprophytic mycobacterium that is used as a surrogate model to study M. tuberculosis genetics and mycobacterial TCSs. We recently demonstrated that $\operatorname{prr} A B$ is not essential in M. smegmatis and that PrAB differentially regulates triacylglycerol biosynthetic genes during ammonium limitation [19]. The inability to generate an $M$. tuberculosis prrAB knockout mutant [15], the high degree of PrrA sequence identity (95\%) between M. tuberculosis and M. smegmatis, and the presence of over 2000 homologous genes
( $51 \%$ of total genes in M. tuberculosis H37Rv) shared between these species prompted use of the M. smegmatis $\triangle \operatorname{prr} A B$ mutant to better understand PrrAB transcriptional regulatory properties. A comprehensive profiling of the genes and pathways regulated by PrAB in M. smegmatis would provide insights into the genetic adaptations that occur during $M$. tuberculosis infection and open new avenues for discovering novel therapeutic targets to treat tuberculosis.

In this study, we used RNA-seq-based transcriptomics analysis to obtain a global profile of the genes regulated by PrAB in $M$. smegmatis. We compared the transcriptomic profiles of $M$. smegmatis WT, $\triangle p r r A B$ mutant, and $\operatorname{pr} A B$ complementation strains during midlogarithmic growth under standard laboratory conditions. Genes repressed by PrAB were associated with broad aspects of metabolism and components of the $\mathrm{F}_{1} \mathrm{~F}_{0}$ ATPase, while PrrAB induced genes involved in oxidoreductase activity, respiration, hypoxic response, and ion homeostasis. These data provide seminal information into the transcriptional regulatory properties of the mycobacterial PrAB TCS and how PrrAB may be controlling molecular processes important in $M$. tuberculosis and other mycobacteria.

\section{Results}

\section{Phylogenetic analyses of PrrA and PrrB in mycobacteria}

Since $\operatorname{pr} A B$ orthologues are present in all mycobacterial species and $\operatorname{pr} A B$ is essential for viability in $M$. tuberculosis [15], it is reasonable to believe that PrAB fulfills important regulatory properties in mycobacteria. We therefore questioned the evolutionary relatedness or distance between PrrA and PrrB proteins in mycobacteria. The M. tuberculosis $\mathrm{H} 37 \mathrm{Rv}$ and M. smegmatis $\mathrm{mc}^{2} 155$ PrrA and PrrB amino acid sequences share 93 and $81 \%$ identity, respectively. Maximum-likelihood phylogenetic trees, based on PrrA (Fig. 1a) and PrrB (Fig. 1b) multiple sequence alignments, were generated. Using the Gupta et al. [20] recent reclassification of mycobacterial species, the results suggested that, with a few exceptions, PrrA and PrrB evolved with specific mycobacterial clades (Fig. 1). While subtle differences in the PrrA or PrrB sequences may represent evolutionary changes as mycobacterial species of the same clade adapted to similar environmental niches, additional experiments are needed to determine if $\operatorname{prr} A B$ is essential in other pathogenic mycobacteria.

We next questioned if the distinct phylogenetic separations between clades could be mapped to specific PrrA or PrrB amino acid residues. We separately aligned mycobacterial PrrA and PrrB sequences in JalView using the default MUSCLE algorithm [21]. Within species of the Abscessus-Chelonae clade, two unique PrrA signatures were found: asparagine and cysteine substitutions relative to serine 38 (S38) and serine 49 (S49), 


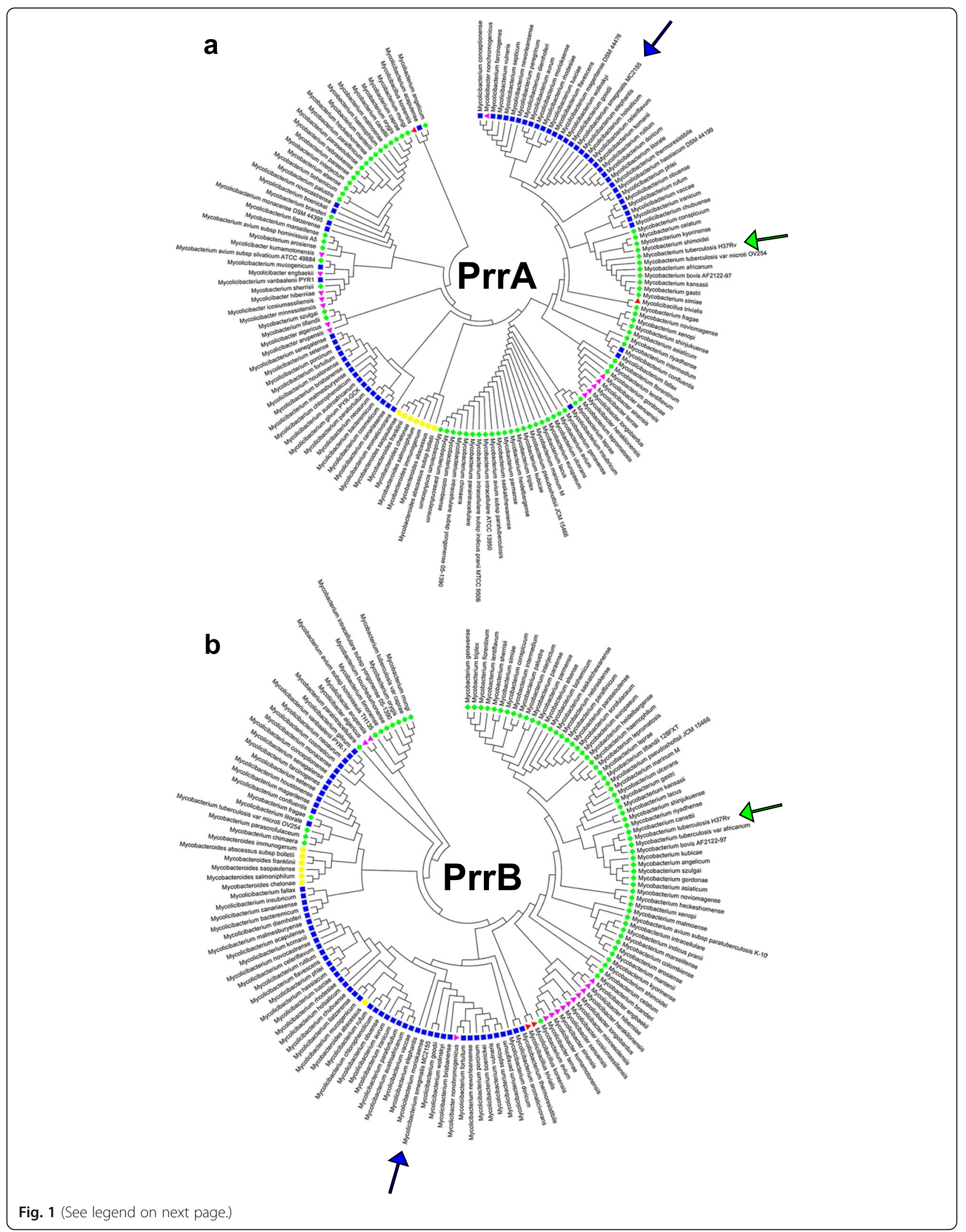


(See figure on previous page.)

Fig. 1 Maximum-likelihood phylogenetic analyses of mycobacterial (a) PrrA and (b) PrrB sequences based on the recent reclassification of mycobacterial species by Gupta et al. [20]. Blue squares, Fortuitum-Vaccae clade. Red triangles, Trivale clade. Green diamonds, Tuberculosis-Simiae clade. Yellow circles, Abscessus-Chelonae clade. Purple triangles, Terrae clade. M. smegmatis mc ${ }^{2} 155$ and M. tuberculosis H37Rv are indicated by blue and green arrows, respectively. PrrA and PrrAB sequences were aligned using default MUSCLE algorithms [21] and phylogenetic tree was generated in MEGA 7 [22]

respectively, of the $M$. smegmatis PrrA sequence (See Additional file 1: Figure S1). These Abscessus-Chelonae clade PrrA residues were not found at similar aligned sites in other mycobacteria (See Additional file 1: Figure S1). Similarly, members of the Abscessus-Chelonae clade (except Mycobacteriodes abscessus) harbored unique amino acid substitutions in PrrB, including glutamate, valine, lysine, aspartate, lysine, and valine corresponding to threonine 42 (T42), glycine 67 (G67), valine 90 (V90), methionine 318 (M318), alanine 352 (A352), and arginine (R371), respectively, of the $M$. smegmatis PrrB sequence (See Additional file 1: Figure S2).

\section{Transcriptomics analysis of the $M$. smegmatis WT, $\triangle p r r A B$} mutant, and complementation strains

We previously generated an $M$. smegmatis $\mathrm{mc}^{2} 155$ prrAB deletion mutant ( $\mathrm{mc}^{2} 155:: \triangle p r r A B$; FDL10) and its complementation strain $\left(\mathrm{mc}^{2} 155: \triangle \operatorname{prr} A B:: \operatorname{prr} A B\right.$; FDL15) [19]. Since the $\operatorname{pr} A B$ regulon and the environmental cue which stimulates PrAB activity are unknown, a global transcriptomics approach was used to analyze differential gene expression in standard laboratory growth conditions. RNA-seq was used to determine transcriptional differences between the $\triangle \operatorname{prr} A B$ mutant, $\mathrm{mc}^{2} 155$, and the complementation strains during mid exponential growth, corresponding to an $\mathrm{OD}_{600}$ of $\sim 0.6$ (See Additional file 1: Figure S3), in supplemented Middlebrook 7H9 (M7H9) broth. Total RNA was isolated from three independent, biological replicates of each $M$. smegmatis strain. Based on multidimensional scaling (MDS) plot, one $\mathrm{mc}^{2} 155$ biological replicate was deemed an outlier and excluded from subsequent analyses (details in Methods, see Additional file 1: Figure S4). Principal component analysis of the global expression patterns of the samples demonstrated that samples from the $\mathrm{mc}^{2} 155$ and FDL15 complementation strains clustered together, apart from those of the FDL10 $\triangle \operatorname{prr} A B$ strain with the majority of variance occurring along PC1 (See Additional file 1: Figure S5), indicating complementation with ectopically-expressed $\operatorname{pr} A B$ in the $\triangle \operatorname{prr} A B$ background.

\section{Identifying the PrrAB regulon}

To identify differentially-expressed genes (DEGs), pairwise comparisons of normalized read counts between the $\triangle \operatorname{prr} A B$ mutant and WT (FDL10 vs. $\mathrm{mc}^{2} 155$ ) as well as the $\triangle \operatorname{prr} A B$ mutant and $\operatorname{pr} A B$ complementation (FDL10 vs. FDL15) datasets were performed using
EdgeR. Deletion of prrAB resulted in induction of 95 genes and repression of 72 genes $(q<0.05)$, representing 167 transcriptional targets (Fig. 2a) that are repressed and induced, respectively, by PrAB in the WT background (Fig. 2c). Less conservative comparisons revealed 683 DEGs $(p<0.05)$ between the WT and $\triangle p r r A B \mathrm{mu}-$ tant strains (See Additional file 1: Figure S6a). Between the $\triangle \operatorname{prr} A B$ complementation and $\operatorname{prr} A B$ mutant strains, 67 DEGs $(q<0.05)$ were identified (Fig. 2b), representing 35 repressed and 32 induced genetic targets by the complementation of PraB (Fig. 2c), while less conservative comparisons $(p<0.05)$ revealed 578 DEGs (See Additional file 1: Figure S6a). Overall, pair-wise DEG analyses revealed that during mid-logarithmic $M$. smegmatis growth, PraB regulates transcription through a relatively balanced combination of gene induction and repression. In addition, comparison between the two DEG sets (i.e., for $\mathrm{mc}^{2} 155$ vs. FDL10 and FDL15 vs. FDL10) datasets revealed 40 (Fig. 2e) and 226 (See Additional file 1: Figure S6b) overlapping DEGs at the significance levels of $q<0.05$ and $p<0.05$, respectively. Hierarchical clustering with the overlapping DEGs further illustrated that gene expression changes induced by the $\operatorname{prr} A B$ deletion were partially recovered by $\operatorname{pr} A B$ complementation (Fig. 2d). We randomly selected six DEGs for qRT-PCR analyses and verified the RNA-seq results for five genes in both the FDL10 vs. mc $^{2} 155$ and FDL10 vs. FDL15 comparisons (See Additional file 1: Figure S7). [See Additional file 2 for a complete list of DEGs between all pairwise comparisons.]

\section{Gene ontology and clustering analyses}

To infer function of the genes regulated by PrAB, enrichment of gene ontology (GO) terms (biological processes and molecular functions) in the DEGs of the $\mathrm{mc}^{2} 155$ vs. FDL10 comparison was assessed by the DAVID functional annotation tool (See Additional file 3 for a complete list of functional annotations returned from the DAVID results). The two sets of DEGs from the $\mathrm{mc}^{2} 155$ vs. FDL10 comparison (See Additional file 1: Figure S6) were examined. In general, genes repressed by PrAB were associated with numerous metabolic processes (Fig. 3a) and nucleotide binding (Fig. 3b), while PrAB-induced genes were associated with ion or chemical homeostasis (Fig. 3c) and oxidoreductase, catalase, and iron-sulfur cluster binding activities (Fig. 3d). Similar GO enrichment terms in the two group comparisons 

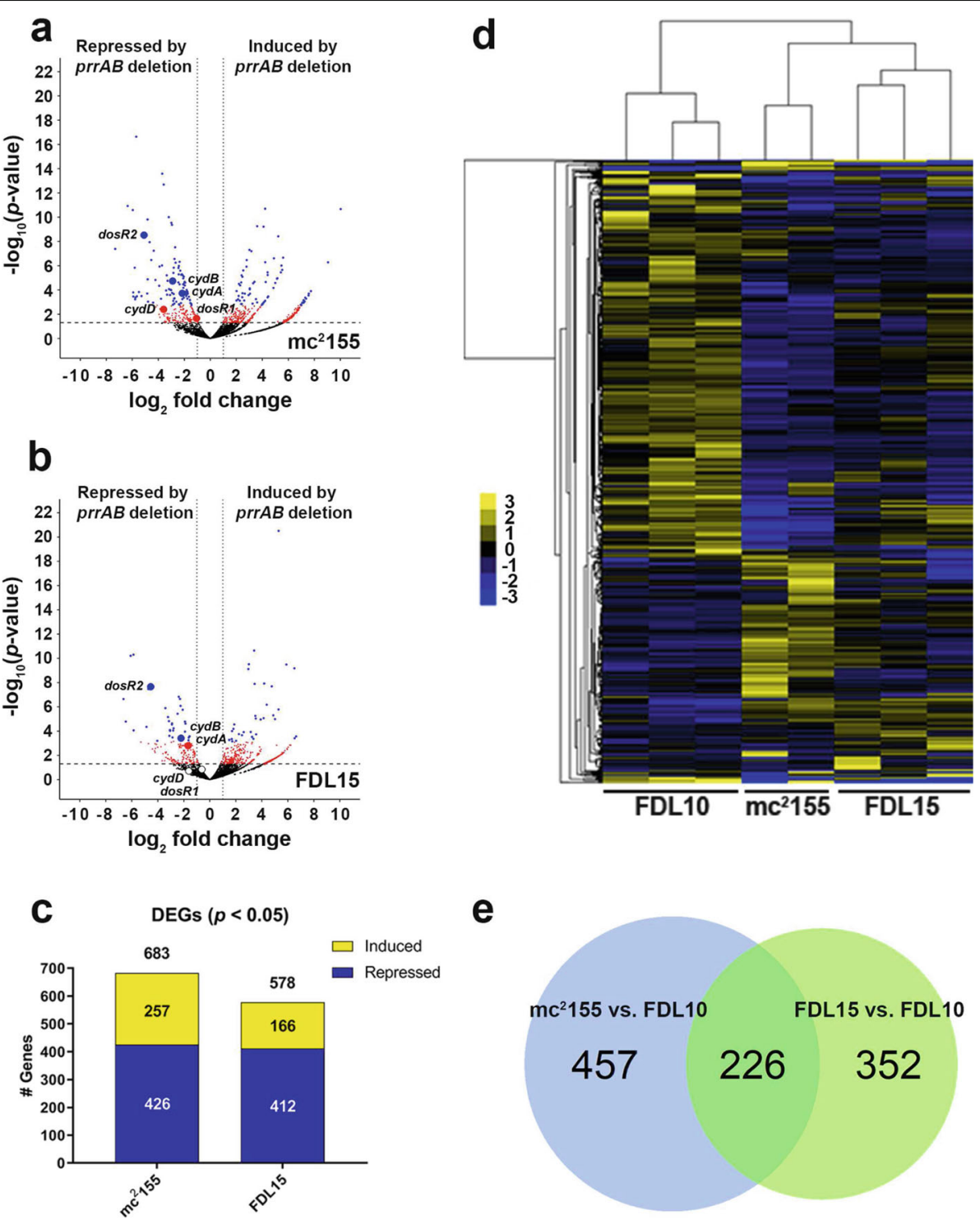

Fig. 2 Global DEG profiles $(q<0.05)$ between the $\mathrm{mc}^{2} 155$ vs. FDL10 and FDL15 vs. FDL10 RNA-seq comparisons. Volcano plots of (a) FDL10 vs. $m^{2} 155$ and (b) FDL10 vs. FDL15 group comparisons with red and blue dots representing differentially-expressed genes with $p<0.05$ and $q<0.05$, respectively. The horizontal hatched line indicates $p=0.05$ threshold, while the left and right vertical dotted lines indicate $\log _{2}$ fold change of -1 and +1 , respectively. c Repressed (blue) and induced (yellow) DEGs $(q<0.05)$ in $\mathrm{mc}^{2} 155$ (WT) and FDL15 (prrAB complementation strain) compared to the FDL10 $\triangle$ prrAB mutant. $\mathbf{d}$ Average hierarchical clustering (FPKM + 1) of individual RNA-seq sample replicates. e Venn diagrams indicating 40 overlapping DEGs $(q<0.05)$ between $\mathrm{mc}^{2} 155$ vs. FDL10 (WT vs. $\triangle$ prrAB mutant) and FDL15 vs. FDL10 (prrAB complementation strain vs. $\triangle p r r A B$ mutant) strain comparisons

$\left(\mathrm{mc}^{2} 155\right.$ vs. FDL10 and FDL15 vs. FDL10) suggested evidence of genetic complementation (Fig. 3; Additional file 1: Figure S8). GO term enrichment was also found for metabolism, nucleotide binding, oxidoreductase, and catalase activity, based on conservative $(q<0.05)$ DEG comparisons (See Additional file 1: Figures S9 and S10). The GO enrichment analyses suggested that during $M$. smegmatis exponential growth in M7H9 medium, PrrAB negatively regulates genes associated with diverse components of metabolic and biosynthetic processes and positively regulates expression of genes participating in respiration $\left(q c r A, c y d A\right.$, and $c y d B$ ), ion transport (via the $\mathrm{F}_{1} \mathrm{~F}_{0}$ ATPase), redox mechanisms, and recognition of environmental signals (dosR2) (Fig. 3; Additional file 1: Figures S8, S9, and S10).

Classification of genes $(q<0.05)$ based on clusters of orthologous groups (COGs) analyses were then performed using the online eggNOG mapper program. Of all COG categories in each gene list, 32\% $(n=22)$ and $24 \%(n=20)$ of genes repressed or induced by PrrAB, 


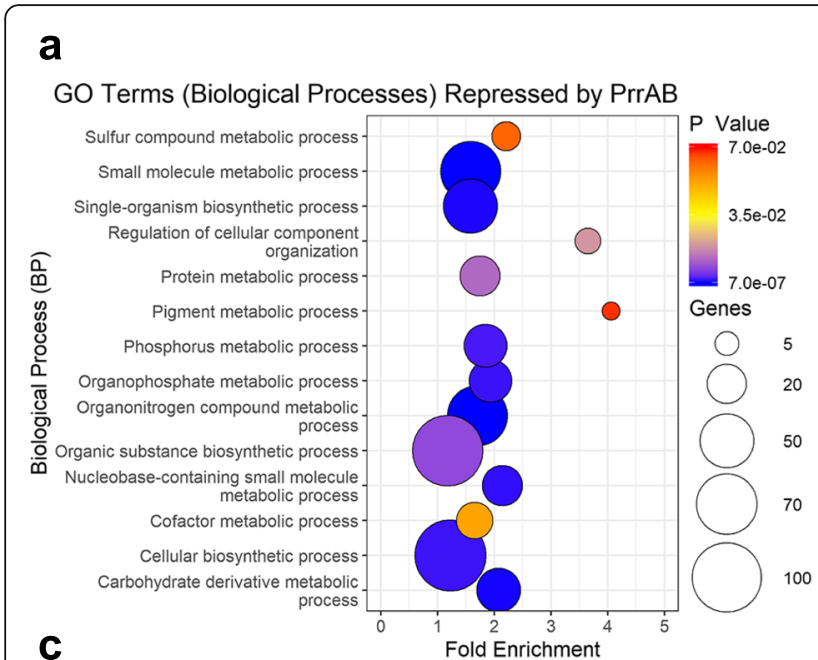

C

GO Terms (Biological Processes) Induced by PrrAB

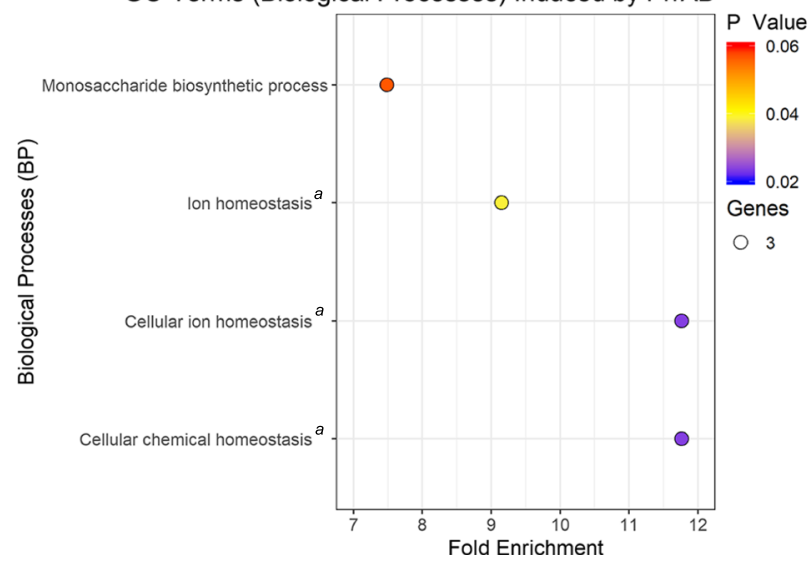

b

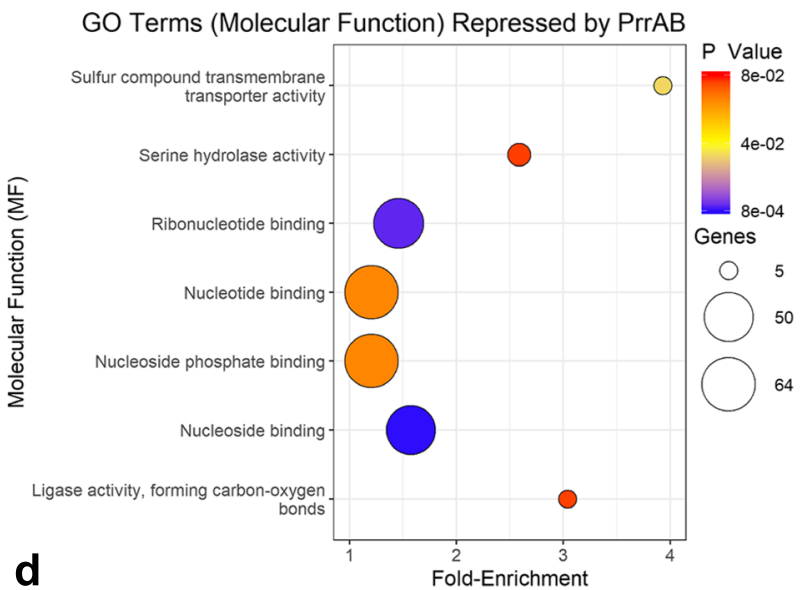

GO Terms (Molecular Function) Induced by PrrAB

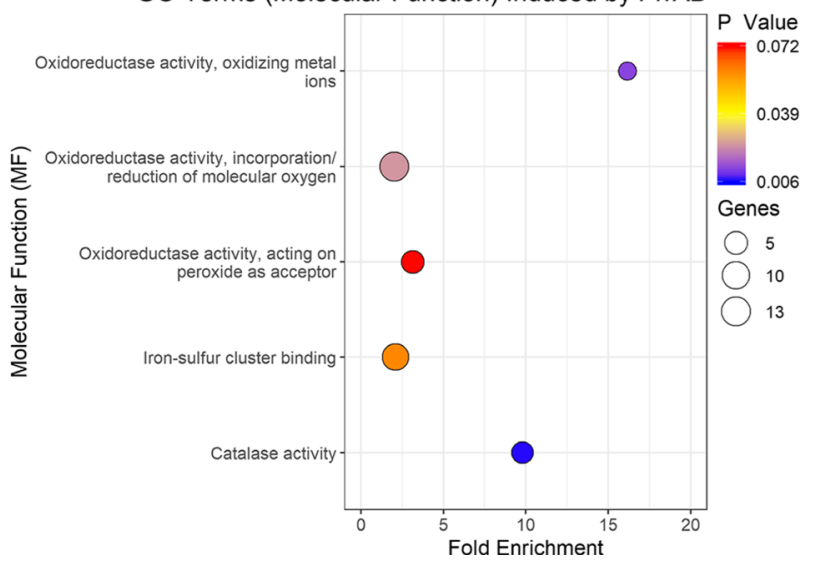

Fig. $3 \mathrm{GO}$ term enrichment associated with DEGs $(p<0.05)$ that are $(\mathbf{a}, \mathbf{b})$ repressed $(\mathbf{c}, \mathbf{d})$ or induced by PrrAB in the WT background. GO terms categorized by $(\mathbf{a}, \mathbf{c})$ biological processes (BP) or (b, d) molecular function (MF). ${ }^{a} \mathrm{GO}$ terms share a common set of genes: MSMEG 3564, MSMEG 6422, and MSMEG 6467

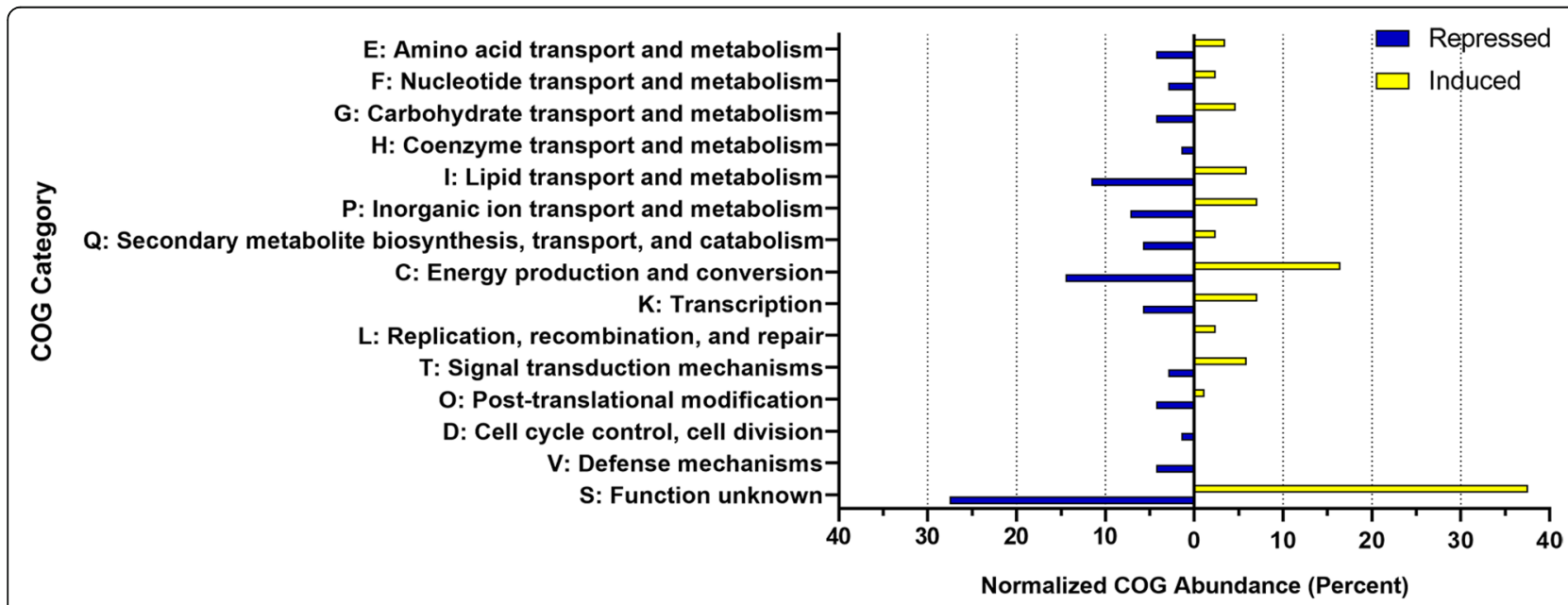

Fig. 4 COG analysis of DEGs $(q<0.05)$ induced (yellow) or repressed (blue) by PrrAB in the WT background. COGs from each category were normalized to represent the percent abundance of each category to all COGs returned in the induced or repressed analyses, respectively 
respectively, participate in diverse aspects of metabolism (Fig. 4), thus corroborating the GO results. Of the COG categories induced by $\operatorname{Pr} A B, 17 \%(n=14)$ were associated with energy production and conversion (COG Category $\mathrm{C}$ ). The relatively even proportions of COG categories associated with PrAB-induced and repressed genes (Fig. 4) suggest that this TCS, as both transcriptional activator and repressor, fine-tunes diverse cellular functions to maximize and/or optimize growth potential during exponential replication.

PrrAB regulates dos $R$ expression in $M$. smegmatis

Differential expression analysis revealed significant repression of MSMEG 5244 and MSMEG 3944, two orthologues of the $\operatorname{dos} R(\operatorname{dev} R)$ response regulator gene, in the $\triangle \operatorname{prr} A B$ mutant strain (Fig. 2a). In $M$. tuberculosis, the hypoxia-responsive DosRS (DevRS) TCS (along with the DosT histidine kinase) induces transcription of $\sim 50$ genes that promote dormancy and chronic infection [23]. Here, we designate MSMEG 5244 as dosR1 (due to its genomic proximity to dosS) and MSMEG 3944 as dosR2. Among the $25 \mathrm{M}$. smegmatis homologues of the $M$. tuberculosis DosRS regulon genes, 7 genes were differentially expressed (+ 2 -fold changes, $q<0.05)$ in pair-wise comparisons among the three strains (Fig. 5 and Additional file 4). Importantly, each of these $M$. smegmatis DosRS regulon homologues were induced by PrAB in the WT and complementation backgrounds, corroborating the activity of the DosR as a positive transcriptional regulator [23].

PrrAB contributes to $M$. smegmatis adaptation to hypoxia The cytochrome $b d$ oxidase respiratory system is a highaffinity terminal oxidase that is important for $M$. smegmatis survival under microaerophilic conditions [24]. Because the $c y d A, c y d B$, and $c y d D$ genes were repressed

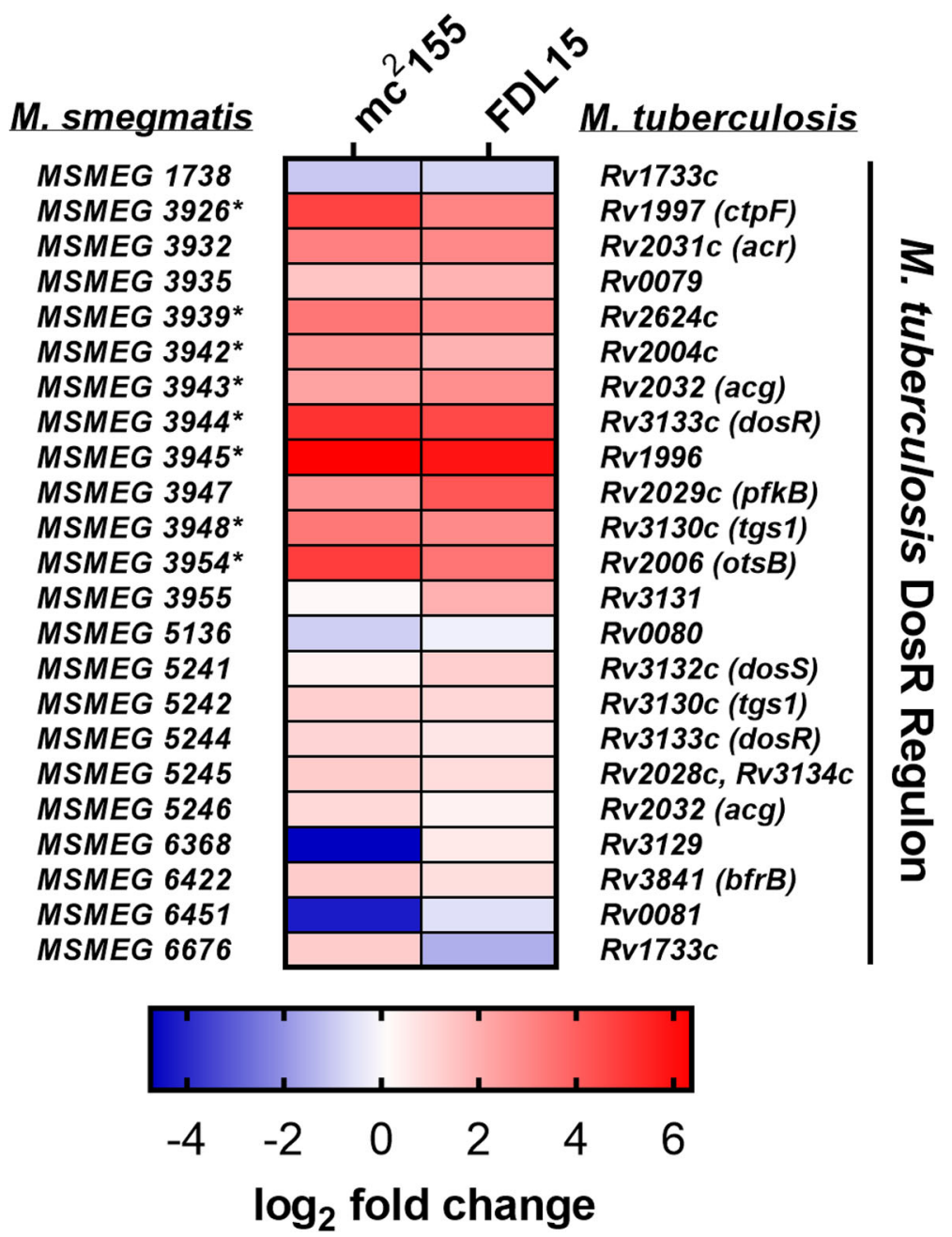

Fig. 5 M. smegmatis PrrAB regulates dormancy-associated genes of the DosR regulon. Heatmap of M. smegmatis RNA-seq DEGs associated with M. tuberculosis dosR regulon homologues. Color bar indicates $\log _{2}$ fold change values corresponding to $m c^{2} 155$ vs. FDL10 (left tiles) and FDL15 vs. FDL10 (right tiles) DEGs. M. smegmatis genes differentially regulated $(q<0.05)$ are denoted by asterisks 
in the $\triangle \operatorname{prr} A B$ mutant during aerobic growth (Fig. 2a; Additional file 2), we questioned if the $\triangle \operatorname{prr} A B$ mutant was more sensitive to hypoxia than the WT strain. Compared to WT and the prrAB complementation strains, the $\triangle \operatorname{prr} A B$ mutant exhibited reduced viability (See Additional file 1: Figure S11a) and produced smaller colonies (See Additional file 1: Figure S11b) after 24h hypoxia exposure. In contrast, cell viability and colony sizes were similar for all strains cultured under aerobic growth conditions (See Additional file 1: Figure S11).

Next, we questioned if differential expression of $c y d A$, $c y d B$, and $c y d D$ correlated with growth deficiencies in the $\triangle \operatorname{prr} A B$ mutant during hypoxia. We compared transcriptional profiles of $c y d A, c y d B$, and $c y d D$ by qRT-PCR from each strain incubated in M7H9 broth under hypoxic and aerobic conditions for $24 \mathrm{~h}$. After $24 \mathrm{~h}$ hypoxia, $c y d A$ and $\operatorname{dos} R 2$ expression was significantly decreased approximately 100 -fold and 10 -fold, respectively, in the $\triangle \operatorname{prr} A B$ mutant relative to the WT strain (Fig. 6a, e). Expression levels of $c y d A$ and $c y d B$ were significantly reduced in the $\triangle \operatorname{prr} A B$ mutant relative to the WT strain during aerobic growth (Fig. 6a, b). Furthermore, both $\operatorname{dos} R 1$ and $\operatorname{dos} R 2$ were significantly downregulated in the $\triangle \operatorname{prr} A B$ mutant under aerobic conditions (Fig. 6d, e), further verifying the RNA-seq data (Additional file 2) and PrAB-mediated regulation in both oxygen-rich and oxygen-poor environmental conditions.

The $\triangle p r r A B$ mutant is hypersensitive to cyanide exposure Cyanide is a potent inhibitor of the $a a_{3}$ cytochrome c oxidase in bacteria. Conversely, cytochrome $b d$ oxidases in Escherichia coli [25], Pseudomonas aeruginosa [26], some staphylococci [27], and M. smegmatis [24] are relatively insensitive to cyanide inhibition. In the absence of alternative electron acceptors (e.g., nitrate and fumarate), aerobic respiratory capacity after cyanide-mediated inhibition of the $M$. smegmatis $a a_{3}$ terminal oxidase would be provided by the cytochrome $b d$ terminal oxidase (CydAB). Because $c y d A, c y d B$, and $c y d D$ were significantly repressed in the $\triangle \operatorname{prr} A B$ mutant (Fig. 2a), as were most subunits of the

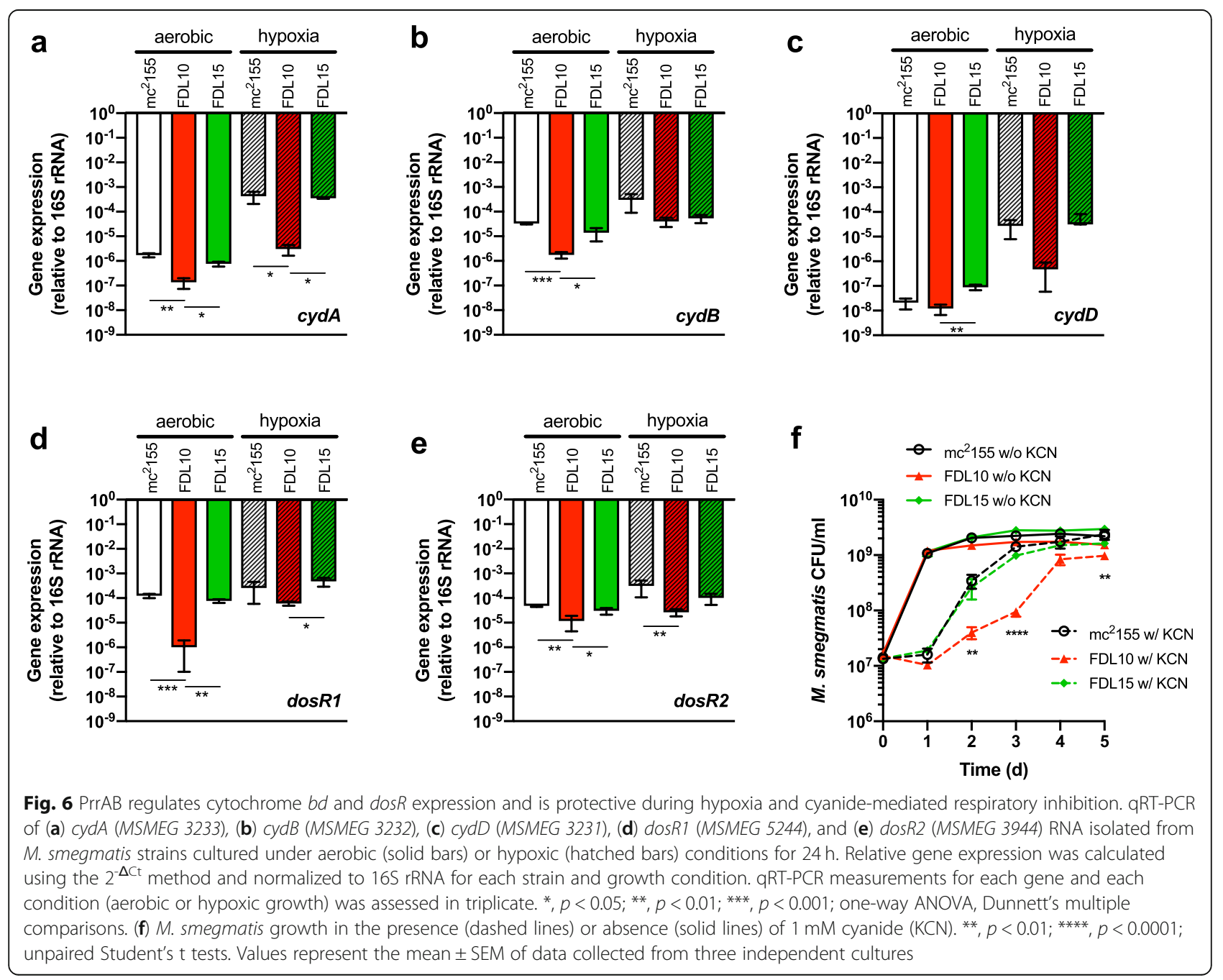


cytochrome c $b c_{1}-a a_{3}$ respiratory oxidase complex (See Additional file 2), we hypothesized that the $\triangle \operatorname{prr} A B$ mutant would be hypersensitive to cyanide relative to the WT and complementation strains. Cyanide inhibited all three strains during the first 24h (Fig. 6f). While the WT and complementation strains entered exponential growth after $24 \mathrm{~h}$ of cyanide exposure, the $\triangle \operatorname{prr} A B$ mutant exhibited significantly delayed and slowed growth between 48 and $72 \mathrm{~h}$ (Fig. 6f). These data demonstrated that the $\triangle \operatorname{prr} A B$ mutant strain had defects in alternative cytochrome $b d$ terminal oxidase pathways, further supporting that genes controlling cytochrome c $b c_{1}$ and $a a_{3}$ respiratory oxidases are induced by PraB.

\section{PrrAB positively regulates ATP levels}

KEGG pathway analysis of DEGs $(p<0.05)$ induced by PrrAB revealed oxidative phosphorylation as a significantly enriched metabolic pathway (Additional file 3 ; enrichment $=3.78 ; p=0.017$ ). Further examination of the RNA-seq data generally revealed that genes of the terminal respiratory complexes (cytochrome c $b c_{1}-a a_{3}$ and cytochrome $b d$ oxidases) were induced by PrrAB, whereas $\mathrm{F}_{1} \mathrm{~F}_{0}$ ATP synthase genes were repressed by PrrAB (Fig. 7a). Therefore, we hypothesized that ATP levels would be greater in the $\triangle \operatorname{prr} A B$ mutant relative to the WT and complementation strains despite the apparent downregulation of terminal respiratory complex genes (except $c t a B$ ) in the $\triangle p r r A B$ mutant (Fig. 7a). While viability was similar between strains at the time of sampling (Fig. 7b), ATP levels ([ATP] pM/CFU) were 36 and $76 \%$ in the $\triangle p r r A B$ mutant and complementation strains, respectively, relative to the WT strain (Fig. 7c). Ruling out experimental artifacts, we confirmed sufficient cell lysis with the BacTiter-Glo reagent (See

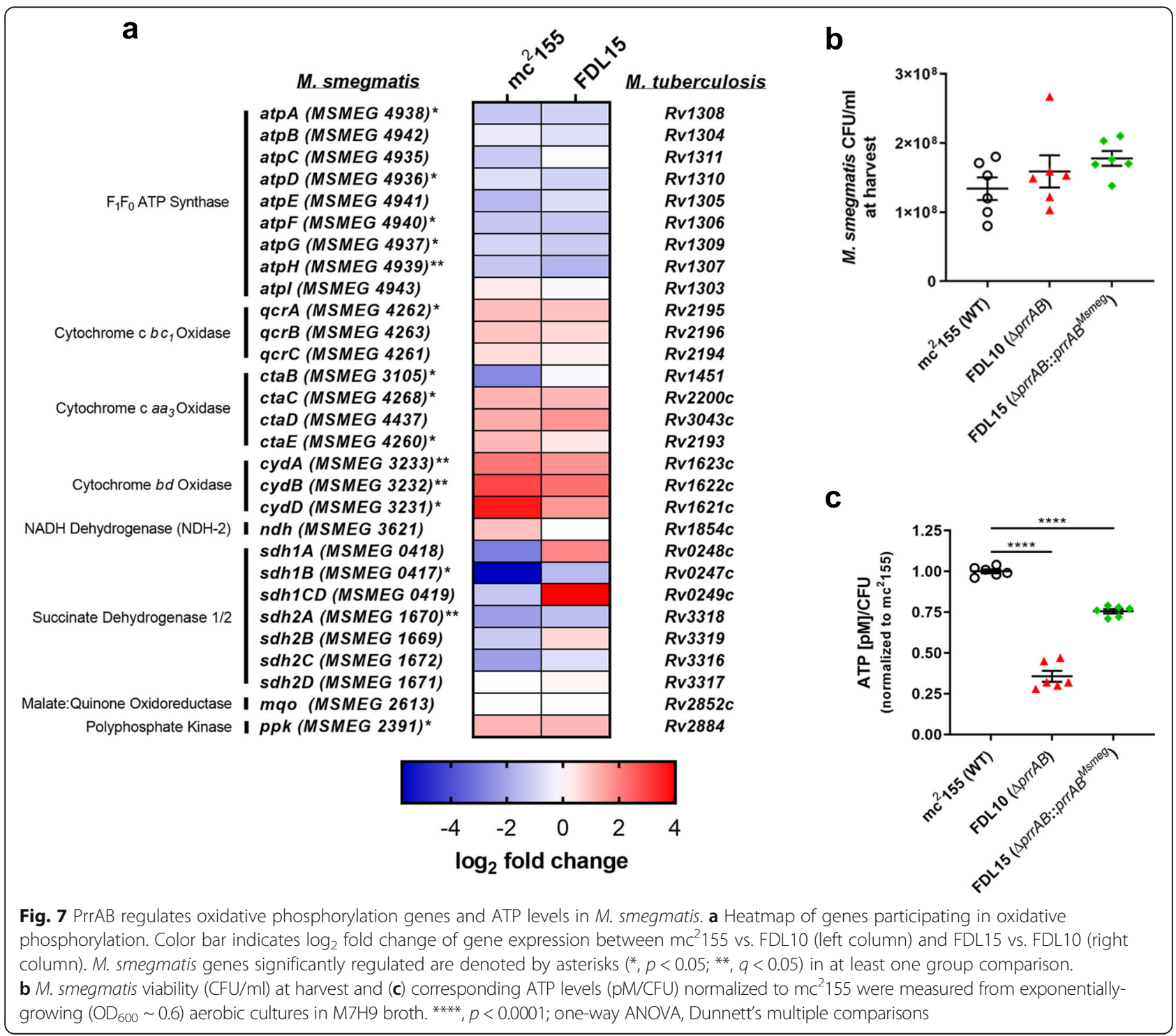


Methods) and that normalized extracellular ATP in cellfree supernatants were similar to intracellular ATP levels (See Additional file 1: Figure S12). These data suggested that PrAB positively regulates ATP levels during aerobic logarithmic growth, although $\operatorname{pr} A B$ complementation did not fully restore ATP to WT levels (Fig. 7c). Additionally, ATP levels correlated with PrrAB induction of respiratory complex genes rather than PrrABmediated repression than $\mathrm{F}_{1} \mathrm{~F}_{0}$ ATP synthase genes (Fig. 7a). To verify the RNA-seq data which indicates PrrAB repression of nearly all $\mathrm{F}_{1} \mathrm{~F}_{0}$ ATP synthase genes (Fig. 7a), we directly measured transcription of three genes in the atp operon: atpC (MSMEG 4935), atpH (MSMEG 4939), and atpI (MSMEG 4943).

The qRT-PCR results revealed that PrrAB represses $\operatorname{atp} C, \operatorname{atpH}$, and $\operatorname{atpI}$ in the WT and $\operatorname{pr} A B$ complementation strains (See Additional file 1: Figure S13).

\section{Discussion}

TCSs provide transcriptional flexibility and adaptive responses to specific environmental stimuli in bacteria [28]. The mycobacterial PrAB TCS is conserved across most, if not all, mycobacterial lineages and is essential for viability in $M$. tuberculosis [15], thus representing an attractive therapeutic target [17]. Here, we use an $M$. smegmatis $\triangle p r r A B$ mutant [19] as a surrogate to provide insights into the essential nature and regulatory properties associated with the PrAB TCS in M. tuberculosis. Our rationale for this approach is founded on the high degree of identity between the M. smegmatis and $M$. tuberculosis PrrA and PrB sequences, including 100\% identity in the predicted DNA-binding recognition helix of PrrA (See Additional file 1: Figure S14) [29].

Using BLAST queries of M. smegmatis PrrA and PrrB against 150 recently reclassified mycobacterial species, as proposed by Gupta et al. [20], all fully-sequenced mycobacterial genomes harbored $\operatorname{prr} A$ and $\operatorname{prr} B$ homologues, implying strong evolutionary conservation for the PraB TCS. Likely due to the incomplete genomic sequences [20], prrA was not found in Mycobacterium timonense and Mycobacterium bouchedurhonense genomes, while a prrB homolog was not identified in Mycobacterium avium subsp. silvaticum. Phylogenetic analyses showed that PrrA and PrrB sequences grouped closely, but not perfectly, within members of specific mycobacterial clades (Fig. 1), and members of the Abscessus-Chelonae clade harbored unique PrrA and PrrB amino acid substitutions (See Additional file 1: Figures S1 and S2). While it is unclear if these residues impact Pra or PrrB functionality in the Abscessus-Chelonae clade, it may be possible to develop prr $A B$-based single nucleotide polymorphism genotyping or proteomic technologies for differentiating mycobacterial infections. Multiple sequence alignments of the M. smegmatis and M. tuberculosis PrrA
DNA-binding recognition helices revealed 100\% sequence conservation (See Additional file 1: Figure S14), suggesting a shared set of core genes regulated by PrA in mycobacteria. Incorporation of a global approach, such as ChIPseq, will be valuable for identifying and characterizing the essential genes directly regulated by $\operatorname{Pr} A$ in $M$. tuberculosis and other mycobacterial species.

We used RNA-seq-based transcriptomics analyses to define the $M$. smegmatis PraB regulon during exponential growth under standard laboratory conditions. We showed that in M. smegmatis, PraB deletion led to differential expression of 167 genes $(q<0.05)$, corresponding to $\sim 2 \%$ of chromosomal genes, of which 95 genes are induced and 72 are repressed in the WT background (Fig. 2). Importantly, PrrAB differentially-regulated genes were involved in aerobic and microaerophilic respiration. The cytochrome c terminal oxidase $b c_{1}(q c r C A B)$ and $a a_{3}$ (ctaC) genes are essential in $M$. tuberculosis, but not in $M$. smegmatis, and mutants in the latter species are attenuated during exponential phase growth [30]. If M. tuberculosis PrAB also regulates genes of the cytochrome c $b c_{1}$ and/or $a a_{3}$ respiratory complex, it could partially explain prr $A B$ essentiality.

To corroborate the key findings from comparing the $\triangle \operatorname{prr} A B$ mutant and WT strains, we included the $\operatorname{prr} A B$ complementation strain in our RNA-seq analyses. Of the 683 DEGs $(p<0.05)$ that were affected by the $\triangle p r r A B$ mutation, expression changes of 10 genes were variably reversed in the $\triangle \operatorname{prr} A B$ complementation strain. Induction of the three genes (MSMEG 5659, MSMEG 5660, and $M S M E G$ 5661) adjacent to $\operatorname{pr} A B$ could be related to alteration of regulatory control sequences during generation of the knockout mutation. These results were unlikely due to poor RNA quality, as RNA integrity numbers (RIN) were consistently high (Additional file 5). We previously demonstrated similar prrA transcription and PrrA protein levels in the WT and complementation strains during aerobic mid-logarithmic growth in M7H9 broth [19], similar to the growth conditions employed in this study. The lack of full complementation seen in our RNA-seq results is likely affected by the low number of biological replicates analyzed. Baccerella et al. [31] demonstrated that sample number impacts RNA-seq performance to a greater degree relative to read depth. Although we found only 226 overlapping DEGs $(p<0.05)$ between the $\mathrm{mc}^{2} 155$ vs. FDL10 and FDL15 vs. FDL10 group comparisons, global DEG regulation (i.e., relative ratios of up- or downregulated genes), was similar. In both pairwise comparisons, 32 and $36 \%$ of all DEGs were induced by PrrAB in the WT and complementation backgrounds, respectively, while 68 and 64\% of all DEGs were repressed by PrAB in the WT and complementation backgrounds, respectively. These data indicate that complementation with $\operatorname{prr} A B$ in the deletion background restored global transcriptomic 
profiles to WT levels. Including additional biological replicates will improve the statistical reliability for better comparison of wild-type and complementation strains which, to the best of our knowledge, has not been previously reported in a transcriptomic study.

We found 40 DEGs $(q<0.05)$ that overlapped between the WT vs. $\triangle p r r A B$ mutant and complementation vs. $\triangle \operatorname{prr} A B$ mutant group comparisons (Fig. 2e). In this data set, enrichment analysis for the GO term "response to stimulus" contains genes of the DosR regulon (Additional file 6). A less-conservative approach using 226 overlapping DEGs $(p<0.05)$ revealed enrichment in GO terms related to respiratory pathways and ATP synthesis (Additional file 6), therefore corroborating our phenotypic and biochemical data (Figs. 6 and 7). It is interesting to postulate that these DEGs may accurately represent the PrrAB regulon in $M$. smegmatis under the conditions tested, as they are significantly represented in both WT and complementation strain group comparisons. Future studies are warranted to explore the utility of incorporating sequencing data from both WT and complementation strains to improve the reliability of transcriptomics experiments.

$M$. tuberculosis acclimates to an intramacrophage environment and the developing granuloma by counteracting the detrimental effects of hypoxia [32], nutrient starvation [33], acid stress [34], and defense against reactive oxygen and nitrogen species [35]. Adaptive measures to these environmental insults include activation of the dormancy regulon and upregulation of the highaffinity cytochrome $b d$ respiratory oxidase [35], induction of the glyoxylate shunt and gluconeogenesis pathways [36], asparagine assimilation [37], and nitrate respiration [38]. As a saprophytic bacterium, M. smegmatis could encounter similar environmental stresses as $M$. tuberculosis, despite their drastically different natural environmental niches. Conserving the gene regulatory circuit of the PrrAB TCS for adaptive responses would thus be evolutionarily advantageous.

The hypoxia-responsive DosRS TCS controls the dormancy regulon in both $M$. tuberculosis [23] and $M$. smegmatis [39-41]. The M. smegmatis DosRS TCS regulates dormancy phenotypes similar to $M$. tuberculosis, including upregulation of the dosRS TCS [39], gradual adaptation to oxygen depletion [42], and upregulation of alanine dehydrogenase [43]. DosR is required for optimal viability in $M$. smegmatis after the onset of hypoxia [41]. Our RNA-seq and qRT-PCR data revealed that PrAB induces both $M$. smegmatis dos $R$ homologues (dosR1 and dosR2) during aerobic and hypoxic growth (Additional file 2, Fig. 2a, Fig. 6d, and Fig. 6e). Additionally, the RNA-seq data revealed that PrrAB induces genes associated with the $M$. tuberculosis DosR regulon [23, 44] (Fig. 5). Thus, it is possible that
PrrAB also positively regulates dos $R$ expression in $M$. tuberculosis, which would provide additional mechanisms of dosR control as previously demonstrated with PknB [45], PknH [46], NarL [47], and PhoP [48].

The M. tuberculosis respiration and oxidative phosphorylation pathways have increasingly gained attention as promising anti-tuberculosis therapeutic targets. Bedaquiline (TMC207), a recent FDA-approved mycobacterial $\mathrm{F}_{1} \mathrm{~F}_{0}$ ATP synthase inhibitor, is active against drug-sensitive and drug-resistant $M$. tuberculosis strains $[49,50]$, as is Q203 (telacebec), a cytochrome c $b c_{1}$ inhibitor, which has advanced to Phase 2 clinical trials [51]. Accumulating evidence suggests that the alternative terminal cytochrome $b d$ oxidase system, encoded by the cydABDC genes in $M$. tuberculosis, is important during chronic infection and may represent a novel drug target. $M$. tuberculosis cydA mutants are hypersensitive to the bactericidal activity of bedaquiline [52], suggesting that combined therapeutic regimens simultaneously targeting the $\mathrm{F}_{1} \mathrm{~F}_{0}$ ATP synthase and cytochrome $b d$ oxidase represent promising antituberculosis treatment strategies. Analysis of the DEGs $(p<0.05)$ induced by PraB (Additional file 3$)$ revealed significant enrichment of the oxidative phosphorylation KEGG pathway, including genes encoding the cytochrome c $b c_{1}(q c r A)$, cytochrome c $a a_{3}$ (ctaC, $c t a E)$, and cytochrome $b d(c y d B, c y d D)$ terminal respiratory branches. We showed that the $\triangle \operatorname{prr} A B \mathrm{mu}$ tant was more sensitive to hypoxic stress and cyanide inhibition relative to the WT and complementation strains (See Additional file 1: Figure S11 and Fig. 6), thus corroborating the transcriptomics results. Although $24 \mathrm{~h}$ hypoxia only caused a modest reduction in the $\triangle \operatorname{prr} A B$ mutant after $24 \mathrm{~h}$ hypoxia exposure, relative to the WT and complementation strains, the $\triangle p r r A B$ mutant small colony phenotype indicated a growth defect under these conditions (See Additional file 1: Figure S11). Additionally, qRT-PCR results demonstrated significantly lower expression of $c y d A$ and $\operatorname{dos} R 2$ in the $\triangle p r r A B$ mutant relative to WT during hypoxic growth, further supporting the biological data. The combined results demonstrate that PrrAB contributes to optimal growth during and after hypoxic stress. We recently reported that the $\triangle \operatorname{prr} A B$ mutant is hypersensitive to hypoxia during growth in lowammonium medium [19]. Our current data suggest that the hypoxia growth defect exhibited by the $\triangle \operatorname{prr} A B$ mutant is likely not medium-specific, but rather a global consequence of differential regulation of respiratory and/ or the $d o s R$ regulon genes. Bacterial cytochrome $b d$ oxidases are relatively insensitive to cyanide inhibition compared to the cytochrome $\mathrm{c}$ oxidase respiratory branch [53-55]. Growth of the $\triangle \operatorname{prr} A B$ mutant in the presence of $1 \mathrm{mM}$ potassium cyanide was similar to $M$. smegmatis $c y d A$ mutant growth under similar conditions [24]. Our 
data demonstrates that the M. smegmatis PrAB TCS controls expression of aerobic and microaerophilic respiratory genes. Notably, to date, a master transcriptional regulator of respiratory systems in $M$. tuberculosis has not been discovered.

We found increased expression of the $\mathrm{F}_{1} \mathrm{~F}_{0}$ ATP synthase genes, including $\operatorname{atp} A, \operatorname{atpD}, \operatorname{atpF}, \operatorname{atp} G$, and $\operatorname{atpH}$, in the $\triangle p r r A B$ mutant strain compared to WT (Fig. 7a; Additional file 1: Figure S13 and Additional file 2), leading us to hypothesize that ATP levels would be elevated in the $\triangle \operatorname{prr} A B$ mutant. Conversely, ATP levels were lower in $\triangle p r r A B$ mutant strain compared to the WT and complementation strains (Fig. 7c). Induction of atp genes in the $\triangle \operatorname{prr} A B$ mutant may indicate a compensatory measure to maintain ATP homeostasis due to repression of the $b c_{1}-a a_{3}$ terminal respiratory complex (except $c t a B$ ) and hence, disruption of the transmembrane proton gradient.

Via comprehensive transcriptomics analyses, we demonstrated that PrrAB regulates expression of genes involved in respiration, environmental adaptation, ion homeostasis, oxidoreductase activity, and metabolism in M. smegmatis. The inability to induce transcription of the $c y d A, c y d B$, $c y d D$, dosR1, and $\operatorname{dos} R 2$ genes likely led the $\triangle \operatorname{prr} A B$ mutant to grow poorly after $24 \mathrm{~h}$ hypoxia exposure. An important goal of our RNA-seq study was to provide insight into the essential nature of PrrAB in $M$. tuberculosis using an $M$. smegmatis $\triangle p r r A B$ mutant as a surrogate model while recognizing differences in their natural environmental niches, pathogenic potential, and genetic composition. From a therapeutic perspective, PrrAB could influence the sensitivity of $M$. tuberculosis to Q203 and/or bedaquiline by controlling expression of cytochrome $b d$ oxidase, cytochrome c $b c_{1}$ oxidase, and ATP synthase genes. Furthermore, it remains unknown whether diarylthiazoles directly target PrrB [17] or whether the prrB mutations associated with diarylthiazole resistance are compensatory in nature. Taken together, our study provides seminal information regarding the mycobacterial PrAB TCS regulon as well as a powerful surrogate platform for in-depth investigations of this essential TCS in M. tuberculosis.

\section{Conclusions}

We used RNA-seq-based transcriptomics as an experimental platform to provide insights into the essential $M$. tuberculosis prrAB TCS using an M. smegmatis $\triangle$ prrAB mutant as a genetic surrogate. In M. smegmatis, PrrAB regulates high-affinity respiratory systems, intracellular redox and ATP balance, and the dosR TCS response regulator genes, all of which promote infectious processes in $M$. tuberculosis. Using these results, we may be able to exploit diarylthiazole compounds that putatively target the PrrB histidine kinase as synergistic therapies with bedaquiline. These results are informing the basis of $\operatorname{prr} A B$ essentiality in $M$. tuberculosis and advancing our understanding of regulatory systems that control metabolic, respiration, energygenerating, and dormancy pathways in mycobacteria. Exploitation of PrrAB as a drug target will advance the discovery and development of novel therapeutics to combat the global tuberculosis epidemic.

\section{Methods \\ Bacterial strains and culture conditions}

Genetic construction of the M. smegmatis FDL10 $\triangle$ prrAB deletion mutant and the FDL15 complementation strain was previously described [19]. All M. smegmatis strains $\left(\mathrm{mc}^{2} 155\right.$, FDL10, and FDL15) were routinely cultured in Middlebrook 7H9 broth (pH 6.8) supplemented with 10\% albumin-dextrose-saline (ADS), $0.2 \%$ glycerol $(\mathrm{v} / \mathrm{v})$, and $0.05 \%$ Tween $80(\mathrm{v} / \mathrm{v})$, herein referred to as M7H9. M. smegmatis was incubated on Middlebrook 7H10 agar supplemented with $10 \%$ ADS and $0.5 \%$ glycerol, herein referred to as $\mathrm{M} 7 \mathrm{H} 10$ agar, for $\mathrm{CFU} / \mathrm{ml}$ enumeration.

\section{Hypoxic growth conditions}

$M$. smegmatis strains were initially cultured aerobically in $\mathrm{M} 7 \mathrm{H} 9$ medium at $37^{\circ} \mathrm{C}, 100 \mathrm{rpm}$ to an $\mathrm{OD}_{600} \sim 0.6$. Cells were diluted into fresh, pre-warmed $\mathrm{M} 7 \mathrm{H} 9$ to an $\mathrm{OD}_{600} \sim 0.05$, serially diluted in PBS (pH 7.4), and spotplated onto M7H10 agar. The plates were transferred to a GasPak chamber containing two anaerobic GasPak sachets (Beckon Dickinson, Franklin Lakes, NJ, USA), sealed, and incubated at $37^{\circ} \mathrm{C}$ for $24 \mathrm{~h}$ after the onset of hypoxia $(\sim 6 \mathrm{~h})$, as indicated by decolorization of an oxygen indicator tablet included with the sachet. Plates were then incubated aerobically for an additional $48 \mathrm{~h}$ to allow colony outgrowth. Control plates were cultured under aerobic conditions for $48 \mathrm{~h}$ prior to counting and documenting colonies. Colonies were visualized using a dissecting microscope (Stereomaster, Fisher Scientific). All experiments were performed in triplicate.

\section{Cyanide inhibition assays}

$M$. smegmatis strains were grown in the presence of potassium cyanide $(\mathrm{KCN})$ as described by [24] with modifications. Briefly, cultures were inoculated into prewarmed M7H9 broth to an $\mathrm{OD}_{600} \sim 0.05$ and incubated at $37^{\circ} \mathrm{C}$, $100 \mathrm{rpm}$ for $30 \mathrm{~min}$. KCN, prepared in M7H9 broth, was then added to a final concentration of $1 \mathrm{mM}$ and growth was allowed to resume. Negative control cultures using $\mathrm{M} 7 \mathrm{H} 9$ broth without $\mathrm{KCN}$ addition were performed concurrently. Cultures were grown for $5 \mathrm{~d}$ with samples collected at $24 \mathrm{~h}$ intervals for $\mathrm{OD}_{600}$ measurements and CFU quantitation on M7H10 agar. All experiments were performed in triplicate. 


\section{ATP assays}

M. smegmatis strains were cultured in M7H9 broth at $37^{\circ} \mathrm{C}, 100 \mathrm{rpm}$. Cultures were sampled in $100 \mu \mathrm{l}$ aliquots upon reaching an $\mathrm{OD}_{600} \sim 0.6$, flash-frozen in a dry iceethanol bath, and stored at $-70{ }^{\circ} \mathrm{C}$ for $7 \mathrm{~d}$. Cells were thawed at room temperature and ATP quantification was performed using the BacTiter-Glo kit (Promega, Madison, WI, USA). Fifty $\mu$ l of cells were mixed with equal volumes of BacTiter-Glo reagent in opaque 96-well plates and incubated at room temperature for $5 \mathrm{~min}$. ATP standard curves were included in the same plate. Relative luminescence was measured in a SpectraMax M5 plate reader (Molecular Devices, San Jose, CA, USA). To assess lysis efficiency, viability of all samples was confirmed after both freeze-thaw and processing in the BacTiter-Glo reagent by plating serial dilutions onto M7H10 agar followed by incubation at $37^{\circ} \mathrm{C}$ for $48-72 \mathrm{~h}$. Lysis efficiencies collected from three independent cultures of $\mathrm{mc}^{2} 155$, FDL10, and FDL15 were 99.97\% ( \pm 0.03$), 99.99 \%( \pm 0.04)$, and $99.99 \%( \pm 0.02)$, respectively. Cell viability was quantified for each sample at the time of harvest by plating serial dilutions onto M7H10 agar followed by incubation at $37^{\circ} \mathrm{C}$ for $48 \mathrm{~h}$ before enumerating CFU/ml. Samples for extracellular ATP measurement were collected as described by Hirokana et al. [56]. Briefly, cells were harvested by centrifugation at 10,621 $\mathrm{x} g$ for $2 \mathrm{~min}$ at $4{ }^{\circ} \mathrm{C}$. The supernatant was clarified via $0.22 \mu \mathrm{m}$ filtration, and aliquots $(100 \mu \mathrm{l})$ were flash-frozen in a dry ice-ethanol bath and stored at $-70{ }^{\circ} \mathrm{C}$ until further use. After thawing, ATP was measured using the BacTiter-Glo kit, as described above. Filtered supernatants were spot plated onto $\mathrm{M} 7 \mathrm{H} 10$ agar and incubated at $37^{\circ} \mathrm{C}$ for $3 \mathrm{~d}$ to verify lack of contaminating cells. All strains were analyzed in triplicate with two technical replicates each.

\section{RNA isolation}

For aerobic cultures, $M$. smegmatis strains $\mathrm{mc}^{2} 155$, FDL10, and FDL15 were grown in $30 \mathrm{ml} \mathrm{M7H9} \mathrm{at} 37^{\circ} \mathrm{C}$, $100 \mathrm{rpm}$ until mid-logarithmic phase $\left(\mathrm{OD}_{600} \sim 0.6\right)$. For hypoxic cultures, $M$. smegmatis strains were first grown $\left(\mathrm{OD}_{600} \sim 0.6\right)$ aerobically in M7H9. Each culture $(15 \mathrm{ml})$ was then transferred a fresh tube, and methylene blue $(1.5 \mu \mathrm{g} / \mathrm{ml}$, final concentration) was added as an indicator of $\mathrm{O}_{2}$ depletion. Cultures were incubated in a sealed GasPak chamber containing two anaerobic sachets (Beckon Dickinson, Franklin Lakes, NJ, USA) for $24 \mathrm{~h}$ post-decolorization of the methylene blue in the media. Culture aliquots $(15 \mathrm{ml})$ were harvested by centrifugation at $3441 \mathrm{x} g$ for $10 \mathrm{~min}$ at $4{ }^{\circ} \mathrm{C}$. The supernatant was discarded, and the cell pellet was resuspended in $1 \mathrm{ml}$ TRIzol (Invitrogen), transferred to $2 \mathrm{ml}$ screw cap tubes containing $500 \mathrm{mg}$ of zirconia-silicate beads (0.1-0.15 $\mathrm{mm})$, and placed on ice. Cells were mechanically disrupted $3 \mathrm{X}$ by bead beating (BioSpec Products) at the highest setting for $40 \mathrm{~s}$ and incubated on ice for at least
1 min between disruptions. The cell lysates were incubated at room temperature for $5 \mathrm{~min}$, centrifuged at 13 , $000 \times g$ for $1 \mathrm{~min}$ to separate cell debris, and the supernatant was transferred to a new microcentrifuge tube. Chloroform $(200 \mu \mathrm{l})$ was added, and samples were vortexed for $15 \mathrm{~s}$ followed by $5 \mathrm{~min}$ incubation at $4{ }^{\circ} \mathrm{C}$. The homogenate was centrifuged at 13,000 $\mathrm{x} g$ for $15 \mathrm{~min}$ at $4{ }^{\circ} \mathrm{C}$ and the upper, aqueous phase was transferred to a new microcentrifuge tube. RNA was precipitated with $500 \mu \mathrm{l}$ isopropanol overnight at $4{ }^{\circ} \mathrm{C}$. Total RNA was pelleted by centrifugation at $13,000 \times g$ for $15 \mathrm{~min}$ at $4{ }^{\circ} \mathrm{C}$, and the supernatant was discarded. RNA pellets were washed $2 \mathrm{X}$ with $70 \%$ ethanol and centrifuged at 13,000 $\mathrm{x}$ $g$ for $5 \mathrm{~min}$ at $4{ }^{\circ} \mathrm{C}$ between washes. After evaporation of residual ethanol by air-drying, total RNA was resuspended in $100 \mu \mathrm{l}$ nuclease-free $\mathrm{H}_{2} \mathrm{O}$. Total RNA $(10 \mu \mathrm{g})$ was treated with TURBO-DNase (Invitrogen, Carlsbad, CA) for $20 \mathrm{~min}$ at $37^{\circ} \mathrm{C}$ to degrade residual genomic DNA. RNA samples were purified using the RNeasy Mini Kit (Qiagen, Germany) and eluted in $50 \mu \mathrm{l}$ nuclease-free $\mathrm{H}_{2} \mathrm{O}$. RNA yields were quantified by Nanodrop (Thermo Scientific, Waltham, MA), and quality was assessed by agarose gel electrophoresis and a 2100 Bioanalyzer (Agilent, Santa Clara, CA). RNA (250 ng) was subjected to PCR using primers directed at the $16 S$ rRNA gene to confirm lack of residual genomic DNA.

\section{RNA-seq library preparation}

cDNA was generated from RNA using the Nugen Ovation RNA-seq System via single primer isothermal amplification and automated on the BRAVO NGS liquid handler (Agilent, Santa Clara, CA, USA). cDNA was quantified on the Nanodrop (Thermo Fisher Scientific) and was sheared to approximately $300 \mathrm{bp}$ fragments using the Covaris M220 ultrasonicator. Libraries were generated using the Kapa Biosystem's library preparation kit (Kapa Biosystems, Wilmington, MA, USA). Fragments were end-repaired and A-tailed and individual indexes and adapters (Bioo, catalogue \#520999) were ligated on each separate sample. The adapter-ligated molecules were cleaned using AMPure beads (Agencourt Bioscience/Beckman Coulter, La Jolla, CA, USA), and amplified with Kapa's HIFI enzyme (Kapa Biosystems, Wilmington, MA, USA). Each library was then analyzed for fragment size on an Agilent Tapestation and quantified by qPCR (KAPA Library Quantification Kit, Kapa Biosystems, Wilmington, MA, USA) using Quantstudio 5 (Thermo Fisher Scientific) prior to multiplex pooling.

\section{Sequencing and data processing}

Sequencing was performed on a $1 \times 75$ bp flow cell using the NextSeq500 platform (Illumina) at the ASU Genomics Core facility. The total number of 101,054,986 Illumina NextSeq500 paired-end reads were generated from 
nine RNA samples (i.e., triplicates for each strain). The total number of reads generated for each sample ranged from 7,729,602 to $14,771,490$. RNA-seq reads for each sample were quality checked using FastQC v 0.10 .1 and aligned to the Mycolicibacterium smegmatis MC2155 assembly obtained from NCBI (https://www.ncbi.nlm.nih. gov/assembly/GCF_000015005.1/) using STAR v2.5.1b. Cufflinks v2.2.1 was used to report FPKM (Fragments Per Kilobase of transcript per Million mapped reads) values and the read counts. As a quality check for the biological replicates, overall similarity of gene expression profiles were then assessed by MDS, in which distances correspond to leading log-fold changes between samples. The MDS analysis demarcated clearly one of the three $\mathrm{mc}^{2} 155$ samples as an outlier that did not cluster with the other two $\mathrm{mc}^{2} 155$ samples and the three FDL15 samples (See Additional file 1: Figure S3), and the sample was thus excluded from further analysis. Average genome-wide expression (FPKM) was 6.76 for the WT strain, 5.88 for the $\triangle$ prrAB mutant, and 6.38 for the complementation strain.

\section{Bioinformatics analysis}

Differential expression analysis was performed with EdgeR package from Bioconductor v3.2 in R 3.2.3. EdgeR applied an overdispersed Poisson model to account for variance among biological replicates. Empirical Bayes tagwise dispersions were also estimated to moderate the overdispersion across transcripts. Then, a negative binomial generalized log-linear model was fit to the read counts for each gene for all comparison pairs. For each pairwise comparison, genes with $p$ values $<0.05$ were considered significant and $\log _{2}$-fold changes of expression between conditions $(\log \mathrm{FC})$ were reported. False discovery rate was calculated following the Benjamini and Hochberg procedure [57], the expected proportion of false discoveries amongst the rejected hypotheses.

Principal component analysis (PCA) was done on the scaled data using the prcomp function in R. Clustering analysis was done using Cluster 3.0 software, in which normalized expression (FPKM +1 ) values were $\log _{2}$ transformed and grouped using uncentered Pearson's correlation distance and average linkage hierarchal clustering [58]. Data matrices and tree dendrograms were visualized in Java TreeView. Gene ontology (GO) term enrichment, KEGG pathways, and statistical analyses of differentially expressed genes were performed using the DAVID functional annotation tool (https://david.ncifcrf.gov/summary.jsp). Clusters of orthologous groups (COGs) were obtained by querying DEGs $(q<0.05)$ against the eggNOG Mapper database (http://eggnogdb.embl.de/\#/app/emapper).

\section{Quantitative RT-PCR (qRT-PCR)}

cDNA libraries from each RNA sample (described above) were generated by reverse transcription of $1 \mu \mathrm{g}$ total RNA using the iScript cDNA Synthesis Kit (Bio-Rad, Hercules, CA, USA), according to the manufacturer's instructions. Primer efficiency was validated against 10 -fold dilution standard curves using a cutoff criterion for acceptable efficiency of $90-110 \%$ and coefficient of determination $\left(R^{2}\right) \geq 0.997$. Relative gene expression was calculated using the $2^{-\Delta \mathrm{Ct}}$ or $2^{-\Delta \Delta \mathrm{Ct}}$ method [59], as indicated, and using the $16 S$ gene as an internal normalization reference. The primers used for qRT-PCR are described in Table S1 (See Additional file 1).

\section{Phylogenetic analyses}

The M. smegmatis $\mathrm{mc}^{2} 155 \operatorname{Pr} \mathrm{A}$ and $\operatorname{PrB}$ sequences were separately queried in BLASTp (https://blast.ncbi. nlm.nih.gov/Blast.cgi) against all Mycobacteriacea (taxid: 1762). Sequences corresponding to the revised mycobacterial phylogenetic clade classification [20] were selected for further analysis. When multiple hits were returned from the same species, those corresponding to the lowest E-value were selected for alignment. Compiled PrrA and PrrB sequences were separately aligned in MEGA 7 (https://www.megasoftware.net/) using default MUSCLE algorithms. Maximum-likelihood phylogenetic trees were generated in MEGA 7 and visualized by iTOL [60].

\section{Statistical analyses}

We used one-way ANOVA to assess significant differences in cell viability, qRT-PCR gene expression, and ATP quantification assays. Statistical analyses were performed using GraphPad Prism 7 (GraphPad Software, San Diego, CA) and $p$-values of $<0.05$ were considered statistically significant. For volcano plot data, the $-\log _{10} p$-value of each DEG was plotted against the ratio of the mean $\log _{2}-$ fold change of each differential expressed gene between FDL10 vs. $\mathrm{mc}^{2} 155$ or FDL10 vs. FDL15.

\section{Supplementary information}

Supplementary information accompanies this paper at https://doi.org/10. 1186/s12864-019-6105-3.

Additional file 1. Supplemental figures.

Additional file 2. M. smegmatis DEG data sets.

Additional file 3. DAVID gene ontology results from significant DEGs.

Additional file 4. DosR regulon DEG and comparison of genes betweeen M. smegmatis and M. tuberculosis.

Additional file 5. RNA Bioanalyzer results (RIN numbers and electrophoretic traces).

Additional file 6. DAVID gene ontology results from overlapping DEGs between mc $^{2} 155$ vs. FDL10 and FDL15 vs. FDL10 data sets.

Abbreviations

ADS: Albumin-dextrose-saline; COG: Clusters of orthologous groups; DEG: Differentially expressed gene; FPKM: Fragments per kilobase of transcript per million mapped reads; GO: Gene ontology; KCN: Potassium cyanide; logFC: $\log _{2}$ fold change; M7H9: Middlebrook $7 \mathrm{H} 9$;

MDS: Multidimensional scaling; PCA: Principal component analysis; qRT- 
PCR: Quantitative reverse transcriptase PCR; TCS: Two-component system; WT: Wild-type

\section{Acknowledgements}

We thank Jason Steel and the Genomics Core at the ASU Biodesign Institute for cDNA library preparations and for performing the Illumina sequencing. We thank Yannik Haller for assisting with the qRT-PCR experiments. We also appreciate the critical insight and suggestions from the anonymous reviewers, which ultimately led to an improved manuscript.

\section{Authors' contributions}

SEH conceived and designed the study with JDM. JDM performed the experiments. SY and JGP processed and analyzed the RNA-seq data. JDM and SY performed the bioinformatics analyses. JDM and SEH analyzed the data. JDM, JGP, and SEH wrote the manuscript. All authors read and approved the final manuscript.

\section{Funding}

This work was partially supported by a Potts Memorial Foundation grant to SEH. The Potts Memorial Foundation was not involved in the design of the study, in the collection, analysis, and interpretation of data, or in writing the manuscript.

\section{Availability of data and materials}

The raw Illumina paired-end sequence data for the RNA-seq studies performed in this article are available at the NCBI Sequence Read Archive (SRA) under the BioProject number PRJNA532282 under accession numbers SAMN11393348, SAMN11393349, and SAMN11393350. The assembled genome sequence for Mycolicibacterium smegmatis MC2 155 can be found in the GenBank database under assembly accession GCA_000015005.1.

\section{Ethics approval and consent to participate}

Not applicable.

\section{Consent for publication}

Not applicable.

\section{Competing interests}

The authors declare that they have no competing interests.

\section{Author details}

${ }^{1}$ School of Life Sciences, Arizona State University, Tempe, AZ, USA. ${ }^{2}$ Department of Obstetrics and Gynecology, College of Medicine-Phoenix, University of Arizona, Phoenix, AZ, USA. ${ }^{3}$ Bioinformatics Core, Knowledge Enterprise Development, Arizona State University, Tempe, AZ, USA. ${ }^{4}$ The Biodesign Institute Virginia G. Piper Center for Personalized Diagnostics, Arizona State University, Tempe, AZ, USA. ${ }^{5}$ The Biodesign Institute Center for Immunotherapy, Vaccines and Virotherapy, Arizona State University, Tempe, AZ, USA.

Received: 5 April 2019 Accepted: 13 September 2019 Published online: 07 December 2019

\section{References}

1. Zschiedrich CP, Keidel V, Szurmant H. Molecular mechanisms of twocomponent signal transduction. J Mol Biol. 2016;428(19):3752-75.

2. Krell T. Exploring the (almost) unknown: Archaeal two-component systems. J Bacteriol. 2018:200(7):10.1128.

3. Schaap P, Barrantes I, Minx P, Sasaki N, Anderson RW, Benard M, et al. The Physarum polycephalum genome reveals extensive use of prokaryotic twocomponent and metazoan-type tyrosine kinase signaling. Genome Biol Evol. 2015:8(1):109-25.

4. Liu Z, Yuan L, Sundaresan V, Yu X. Arabidopsis CKI1 mediated twocomponent signaling in the specification of female gametophyte. Plant Signal Behav. 2018;13(10):e1469360.

5. Kumar A, Toledo JC, Patel RP, Lancaster JR Jr, Steyn AJ. Mycobacterium tuberculosis DosS is a redox sensor and DosT is a hypoxia sensor. Proc Nat Acad Sci U S A. 2007;104(28):11568-73.

6. Richmond GE, Evans LP, Anderson MJ, Wand ME, Bonney LC, Ivens A, et al. The Acinetobacter baumannii two-component system AdeRS regulates genes required for multidrug efflux, biofilm formation, and virulence in a strain-specific manner. mBio. 2016:7(2):e00430-16.

7. Gebhardt MJ, Shuman HA. GigA and GigB are master regulators of antibiotic resistance, stress responses, and virulence in Acinetobacter baumannii. J Bacteriol. 2017;199(10):10.1128.

8. Kelliher JL, Radin JN, Kehl-Fie TE. PhoPR contributes to Staphylococcus aureus growth during phosphate starvation and pathogenesis in an environment-specific manner. Infect Immun. 2018;86(10):10.1128.

9. Mehra S, Foreman TW, Didier PJ, Ahsan MH, Hudock TA, Kissee R, et al. The DosR regulon modulates adaptive immunity and is essential for Mycobacterium tuberculosis persistence. Am J Respir Crit Care Med. 2015; 191(10):1185-96

10. Herrera CM, Crofts AA, Henderson JC, Pingali SC, Davies BW, Trent MS. The Vibrio cholerae VprA-VprB two-component system controls virulence through endotoxin modification. mBio. 2014;5(6):10.1128.

11. Srivastava S, Li Z, Ko K, Choudhury P, Albaqumi M, Johnson AK, et al. Histidine phosphorylation of the potassium channel KCa3.1 by nucleoside diphosphate kinase $\mathrm{B}$ is required for activation of KCa3.1 and CD4 T cells. Mol Cell. 2006:24(5):665-75.

12. WHO. Global tuberculosis report 2017. Geneva: The World Health Organization; 2017.

13. Haydel SE, Clark-Curtiss JE. Global expression analysis of two-component system regulator genes during Mycobacterium tuberculosis growth in human macrophages. FEMS Microbiol Lett. 2004;236(2):341-7.

14. Zahrt TC, Deretic V. An essential two-component signal transduction system in Mycobacterium tuberculosis. J Bacteriol. 2000;182(13):3832-8.

15. Haydel SE, Malhotra V, Cornelison GL, Clark-Curtiss JE. The prrAB twocomponent system is essential for Mycobacterium tuberculosis viability and is induced under nitrogen-limiting conditions. J Bacteriol. 2012;194(2):354-61.

16. Ewann $F$, Jackson M, Pethe $K$, Cooper A, Mielcarek N, Ensergueix D, et al. Transient requirement of the PrrA-PrrB two-component system for early intracellular multiplication of Mycobacterium tuberculosis. Infect Immun. 2002;70(5):2256-63.

17. Bellale E, Naik M, BV V, Ambady A, Narayan A, Ravishankar S, et al. Diarylthiazole: an antimycobacterial scaffold potentially targeting PrrB-PrrA two-component system. J Med Chem. 2014;57(15):6572-82.

18. Snapper SB, Melton RE, Mustafa S, Kieser T, Jacobs WR Jr. Isolation and characterization of efficient plasmid transformation mutants of Mycobacterium smegmatis. Mol Microbiol. 1990;4(11):1911-9.

19. Maarsingh JD, Haydel SE. Mycobacterium smegmatis PrrAB two-component system influences triacylglycerol accumulation during ammonium stress. Microbiology. 2018;164(10):1276-88.

20. Gupta RS, Lo B, Son J. Phylogenomics and comparative genomic studies robustly support division of the genus Mycobacterium into an emended genus Mycobacterium and four novel genera. Front Microbiol. 2018;9:67.

21. Edgar RC. MUSCLE: a multiple sequence alignment method with reduced time and space complexity. BMC Bioinformatics. 2004:5:113-2105 -2105-2113.

22. Kumar S, Stecher G, Tamura K. MEGA7: molecular evolutignary genetics analysis version 7.0 for bigger datasets. Mol Biol Evol. 2016:33(7):1870-4

23. Park HD, Guinn KM, Harrell MI, Liao R, Voskuil MI, Tompa M, et al. Rv3133c/ dos $R$ is a transcription factor that mediates the hypoxic response of Mycobacterium tuberculosis. Mol Microbiol. 2003;48(3):833-43.

24. Kana BD, Weinstein EA, Avarbock D, Dawes SS, Rubin H, Mizrahi V. Characterization of the $c y d A B$-encoded cytochrome bd oxidase from Mycobacterium smegmatis. J Bacteriol. 2001:183(24):7076-86.

25. Korshunov S, Imlay KR, Imlay JA. The cytochrome bd oxidase of Escherichia coli prevents respiratory inhibition by endogenous and exogenous hydrogen sulfide. Mol Microbiol. 2016;101(1):62-77.

26. Cunningham L, Pitt M, Williams HD. The cioAB genes from Pseudomonas aeruginosa code for a novel cyanide-insensitive terminal oxidase related to the cytochrome bd quinol oxidases. Mol Microbiol. 1997;24(3):579-91.

27. Voggu L, Schlag S, Biswas R, Rosenstein R, Rausch C, Gotz F. Microevolution of cytochrome bd oxidase in staphylococci and its implication in resistance to respiratory toxins released by Pseudomonas. J Bacteriol. 2006;188(23):8079-86.

28. Calva E, Oropeza R. Two-component signal transduction systems, environmental signals, and virulence. Microb Ecol. 2006;51(2):166-76.

29. Nowak E, Panjikar S, Konarev P, Svergun DI, Tucker PA. The structural basis of signal transduction for the response regulator PrrA from Mycobacterium tuberculosis. J Biol Chem. 2006;281(14):9659-66.

30. Matsoso LG, Kana BD, Crellin PK, Lea-Smith DJ, Pelosi A, Powell D, et al. Function of the cytochrome $b c_{1}-a a_{3}$ branch of the respiratory network in 
mycobacteria and network adaptation occurring in response to its disruption. J Bacteriol. 2005;187(18):6300-8.

31. Baccarella A, Williams CR, Parrish JZ, Kim CC. Empirical assessment of the impact of sample number and read depth on RNA-Seq analysis workflow performance. BMC Bioinformatics. 2018;19(1):423.

32. Aly S, Wagner K, Keller C, Malm S, Malzan A, Brandau S, et al. Oxygen status of lung granulomas in Mycobacterium tuberculosis-infected mice. J Pathol. 2006;210(3):298-305.

33. Schnappinger D, Ehrt S, Voskuil MI, Liu Y, Mangan JA, Monahan IM, et al. Transcriptional adaptation of Mycobacterium tuberculosis within macrophages: insights into the phagosomal environment. J Exp Med. 2003; 198(5):693-704.

34. Rohde KH, Veiga DF, Caldwell S, Balazsi G, Russell DG. Linking the transcriptional profiles and the physiological states of Mycobacterium tuberculosis during an extended intracellular infection. PLoS Path. 2012;8(6):e1002769.

35. Voskuil MI, Visconti KC, Schoolnik GK. Mycobacterium tuberculosis gene expression during adaptation to stationary phase and low-oxygen dormancy. Tuberculosis. 2004;84(3-4):218-27.

36. Timm J, Post FA, Bekker LG, Walther GB, Wainwright HC, Manganelli R, et al. Differential expression of iron-, carbon-, and oxygen-responsive mycobacterial genes in the lungs of chronically infected mice and tuberculosis patients. Proc Natl Acad Sci U S A. 2003;100(24):14321-6.

37. Gouzy A, Larrouy-Maumus G, Bottai D, Levillain F, Dumas A, Wallach JB, et al. Mycobacterium tuberculosis exploits asparagine to assimilate nitrogen and resist acid stress during infection. PLoS Path. 2014;10(2): e1003928.

38. Tan MP, Sequeira P, Lin WW, Phong WY, Cliff P, Ng SH, et al. Nitrate respiration protects hypoxic Mycobacterium tuberculosis against acid- and reactive nitrogen species stresses. PLoS One. 2010;5(10):e13356.

39. Mayuri B. G, das TK, Tyagi JS. Molecular analysis of the dormancy response in Mycobacterium smegmatis: expression analysis of genes encoding the DevR-DevS two-component system, Rv3134c and chaperone alpha-crystallin homologues. FEMS Microbiol Lett. 2002;211(2):231-7.

40. Bagchi G. Mayuri, Tyagi JS. Hypoxia-responsive expression of Mycobacterium tuberculosis Rv3134C and devR promoters in Mycobacterium smegmatis. Microbiology. 2003;149:2303-5.

41. O'Toole R, Smeulders MJ, Blokpoel MC, Kay EJ, Lougheed K, Williams HD. A two-component regulator of universal stress protein expression and adaptation to oxygen starvation in Mycobacterium smegmatis. J Bacteriol. 2003;185(5):1543-54

42. Dick T, Lee BH, Murugasu-Oei B. Oxygen depletion induced dormancy in Mycobacterium smegmatis. FEMS Microbiol Lett. 1998;163(2):159-64.

43. Feng Z, Caceres NE, Sarath G, Barletta RG. Mycobacterium smegmatis Lalanine dehydrogenase (Ald) is required for proficient utilization of alanine as a sole nitrogen source and sustained anaerobic growth. J Bacteriol. 2002; 184(18):5001-10

44. Voskuil MI, Schnappinger D, Visconti KC, Harrell MI, Dolganov GM, Sherman DR, et al. Inhibition of respiration by nitric oxide induces a Mycobacterium tuberculosis dormancy program. J Exp Med. 2003;198(5):705-13.

45. Bae HJ, Lee HN, Baek MN, Park EJ, Eom CY, Ko IJ, et al. Inhibition of the DevSR two-component system by overexpression of Mycobacterium tuberculosis PknB in Mycobacterium smegmatis. Mol Cells. 2017;40(9):632-42.

46. Chao JD, Papavinasasundaram KG, Zheng X, Chavez-Steenbock A, Wang $X$, Lee $\mathrm{GQ}$, et al. Convergence of Ser/Thr and two-component signaling to coordinate expression of the dormancy regulon in Mycobacterium tuberculosis. J Biol Chem. 2010;285(38):29239-46.

47. Malhotra V, Agrawal R, Duncan TR, Saini DK, Clark-Curtiss JE. Mycobacterium tuberculosis response regulators, DevR and NarL, interact in vivo and coregulate gene expression during aerobic nitrate metabolism. J Biol Chem. 2015;290(13):8294-309.

48. Vashist A, Malhotra V, Sharma G, Tyagi JS, Clark-Curtiss JE. Interplay of PhoP and DevR response regulators defines expression of the dormancy regulon in virulent Mycobacterium tuberculosis. J Biol Chem. 2018;293(42): $16413-25$.

49. Diacon AH, Pym A, Grobusch M, Patientia R, Rustomjee R, Page-Shipp L, et al. The diarylquinoline TMC207 for multidrug-resistant tuberculosis. New Engl J Med. 2009;360(23):2397-405.

50. Andries K, Verhasselt P, Guillemont J, Gohlmann HWH, Neefs JM, Winkler H, et al. A diarylquinoline drug active on the ATP synthase of Mycobacterium tuberculosis. Science. 2005;307(5707):223-7.
51. Butler MS, Blaskovich MA, Cooper MA. Antibiotics in the clinical pipeline at the end of 2015. J Antibiot. 2017;70(1):3-24.

52. Berney M, Hartman TE, Jacobs WR Jr. A Mycobacterium tuberculosis cytochrome bd oxidase mutant is hypersensitive to bedaquiline. mBio. 2014;5(4):e01275-14.

53. Megehee JA, Hosler JP, Lundrigan MD. Evidence for a cytochrome bcc- $a a_{3}$ interaction in the respiratory chain of Mycobacterium smegmatis. Microbiology. 2006;152(3):823-9.

54. Hammer ND, Schurig-Briccio LA, Gerdes SY, Gennis RB, Skaar EP. CtaM is required for menaquinol oxidase $a a_{3}$ function in Staphylococcus aureus. mBio. 2016;7(4). https://doi.org/10.1128/mBio.00823-00816.

55. Hirai T, Osamura T, Ishii M, Arai $H$. Expression of multiple $c b b_{3}$ cytochrome $c$ oxidase isoforms by combinations of multiple isosubunits in Pseudomonas aeruginosa. Proc Natl Acad Sci U S A. 2016;113(45):12815-9.

56. Hironaka I, Iwase T, Sugimoto S, Okuda K, Tajima A, Yanaga K, et al. Glucose triggers ATP secretion from bacteria in a growth-phase-dependent manner. Appl Environ Microbiol. 2013;79(7):2328-35.

57. Benjamini $Y$, Hochberg Y. Controlling the false discovery rate: a practical and powerful approach to multiple testing. J Roy Stat Soc Ser B. 1995;57(1):289-300.

58. Eisen MB, Spellman PT, Brown PO, Botstein D. Cluster analysis and display of genome-wide expression patterns. Proc Natl Acad Sci U S A. 1998;95(25): 14863-8.

59. Livak KJ, Schmittgen TD. Analysis of relative gene expression data using real-time quantitative $P C R$ and the $2^{-\Delta \Delta C t}$ method. Methods. 2001;25(4):402-8

60. Letunic I, Bork P. Interactive tree of life (iTOL) v3: an online tool for the display and annotation of phylogenetic and other trees. Nucleic Acids Res. 2016;44(W1):W242-5.

\section{Publisher's Note}

Springer Nature remains neutral with regard to jurisdictional claims in published maps and institutional affiliations.
Ready to submit your research? Choose BMC and benefit from:

- fast, convenient online submission

- thorough peer review by experienced researchers in your field

- rapid publication on acceptance

- support for research data, including large and complex data types

- gold Open Access which fosters wider collaboration and increased citations

- maximum visibility for your research: over $100 \mathrm{M}$ website views per year

At BMC, research is always in progress.

Learn more biomedcentral.com/submissions 